\title{
FIBROADENOMA JUVENIL GIGANTE EM PACIENTE HEBIÁTRICO: RELATO DE CASO
}

Isabella Figueiredo Dias, Leonado Gomes Baldoino, Herbert Hornig, Gabriel Ishibashi Tatibana Kazuo, Samira Monteiro Martins, Rafael da Silva Sá

Universidade do Oeste Paulista - UNOESTE, Faculdade de Medicina, Presidente Prudente, SP. e-mail: isa fdias@hotmail.com

\section{RESUMO}

O fibroadenoma é o tumor de mama mais frequente em mulheres entre 10 a 20 anos, sendo uma neoplasia que se caracteriza por uma multiplicação benigna com crescimento rápido e geralmente em forma de nódulo único. Foi realizado um estudo de caso clínico de uma adolescente de 12 anos e 7 meses de idade, a qual apresentava uma tumoração na mama esquerda, ocupando toda a sua extensão. Quando o tumor for maior que $5 \mathrm{~cm}$ ou pesar mas que $500 \mathrm{~g}$ ou ocupar pelo menos $80 \%$ da mama, é considerado um fibroadenoma juvenil gigante. A extirpação cirúrgica tumoral é a melhor opção tanto para o diagnóstico quanto para o tratamento, devido à necessidade de avaliação histopatológica. É um quadro que necessita de atenção, pois tem a característica de mimetizar outras patologias, especialmente neoplasias malignas, além de ter uma evolução muito rápida.

Palavras-chave: fibroadenoma, mama, neoplasias da mama.

\section{GIANT JUVENILE FIBROADENOMA IN HEBIATRIC PATIENT. A CASE REPORT}

\section{ABSTRACT}

Fibroadenoma is the most frequent breast tumor in women aged 10 to 20 years, a neoplasm characterized by a benign multiplication with rapid growth and usually in the form of a single lump. A clinical case report study was performed on a 12 year and 7 month old girl, who had a left breast tumor, occupying all its extension. When the tumor is larger than $5 \mathrm{~cm}$ or $500 \mathrm{~g}$ or when it occupies at least $80 \%$ of the breast is considered a giant juvenile fibroadenoma. The choice of tumor surgical excision is the best option for both diagnosis and treatment, due to the need for pathologic evaluation. It is a picture that needs attention, since it has the characteristic of mimicking other pathologies, besides having a very rapid evolution.

Keywords: fibroadenoma, breast, breast neoplasms.

\section{INTRODUÇÃO}

O fibroadenoma é o tumor de mama mais frequente em mulheres entre 10 a 20 anos de idade, representando $75 \%$ da totalidade, sendo mais comum em mulheres da raça negra e em asiáticas. Esta neoplasia caracteriza-se por uma multiplicação benigna do componente mesenquimal e epitelial da mama, com crescimento rápido e, geralmente, constitui nódulo único. Nódulos múltiplos uni ou bilaterais são menos comuns $(15 \%$ e $10 \%$ dos casos, respectivamente) $)^{1}$. Os tumores têm tamanho variável, são bem delimitados, indolores, com consistência dura ou elástica e é móvel ${ }^{2}$. Quando apresenta diâmetro maior que $5 \mathrm{~cm}$, peso maior que $500 \mathrm{~g}$ ou ocupa pelo menos $80 \%$ da mama, é considerado como um fibroadenoma juvenil gigante, sendo menos comum, compreendendo de $1-8 \%$ das lesões mamárias na população adolescente $^{3}$. Histologicamente, caracteriza-se 
pelo aumento da celularidade estromal, com padrão de crescimento pericanalicular, hiperplasia dos ductos e fibrose do estroma ${ }^{4,5}$. Os fatores etiológicos não estão totalmente esclarecidos, mas aparentam ser hormônio dependentes ${ }^{5}$. Possíveis fatores incluem trauma, gravidez, lactação, excesso de estrógeno e efeitos dos hormônios da puberdade.

Os nódulos mamários benignos estão entre os $80 \%$ das massas que são palpáveis, tendo o quadro de diagnóstico diferencial muito amplo, incluindo cistos mamários, fibroadenomas, tumores filóides, papilomas, lipomas, hamartomas e adenomas ${ }^{6}$. A diferenciação entre os nódulos sólidos e os císticos é feita através da punção aspirativa com agulha fina ou por ultrassonografia ${ }^{7}$.

O diagnóstico é feito através do exame físico, exames imaginológicos (mamografia, ecografia ou ressonância magnética), sendo o diagnóstico definitivo realizado através do laudo citológico/histopatológico (punção aspirativa por agulha fina/punção por agulha grossa) ${ }^{6}$.

Dentre as possíveis complicações encontram-se: lesões por compressão de tecidos adjacentes, deformação da estrutura mamária, retração mamilar e expansão das veias superficiais $^{8}$. Outras complicações englobam úlceras da pele e estresse psicossocial ${ }^{9}$.

O tratamento constitui-se na exérese completa do tumor ${ }^{10}$. Busca-se a preservação do complexo areolar bem como a simetria mamária ${ }^{1}$. A mastectomia é uma das modalidades de tratamento para fibroadenomas gigantes, mas geralmente reserva-se aos casos incomuns e recorrentes. Nos casos em que se utiliza mastectomia, normalmente as pacientes são submetidas à cirurgia reparadora ${ }^{3}$.

O objetivo deste trabalho é relatar um caso de uma paciente do gênero feminino, de 12 anos e 7 meses de idade, apresentando fibroadenoma juvenil gigante.

\section{RELATO DE CASO}

Paciente de 12 anos e 7 meses de idade, parda, católica, estudante, natural e procedente de Bataguassu - MS, referindo aumento mamário esquerdo abrupto, associado à mastologia acíclica. Menarca aos 11 anos. Ao exame físico, a paciente apresentava mama esquerda com tumoração de grande volume, ocupando toda a extensão mamária esquerda (Figura 1).

A ultrassonografia mamária revelou volumosa formação expansiva, de aspecto sólido, vascularizada, com contornos regulares, ocupando toda a mama esquerda, sendo classificada como BI-RADS categoria 4A. Não foi possível obter uma definição precisa das dimensões da lesão, já que a mesma excedia os limites do transdutor ultrassonográfico. Deste modo, foi realizado biópsia mamária percutânea (punção de mama por agulha grossa), tendo sido encaminhados para o exame anatomopatológico 3 fragmentos filiformes de tecido, medindo entre $2 \times 2 \mathrm{~cm}$ e $1 \times 0,2 \mathrm{~cm}$, de coloração castanho clara e de consistência firme e elástica. A microscopia descartou malignidade, com resultado de hiperplasia ductal sem atipias, ectasias discretas e hiperplasia fibroadiposa do estroma acentuada.

A conduta cirúrgica realizada foi tumorectomia, com incisão via sulco inframamário e o pós-operatório foi sem intercorrências (Figura 2). 0 tumor foi encaminhado para avaliação anatomopatológica, cujo relatório apresentava na macroscopia uma formação nodular, com peso de $530 \mathrm{~g}$, medindo $14 \times 12 \times 7,5 \mathrm{~cm}$ de diâmetro, com superfície externa lobulada, de coloração castanho clara (Figura 3), concluindo pelo diagnóstico de fibroadenoma mamário juvenil.

Paciente em acompanhamento semestral, sem recidiva e assintomática (Figura 4).

O estudo foi avaliado e aprovado pelo Comitê de Ética em Pesquisa da UNOESTE (CAAE no 98166918.5.0000.5515).

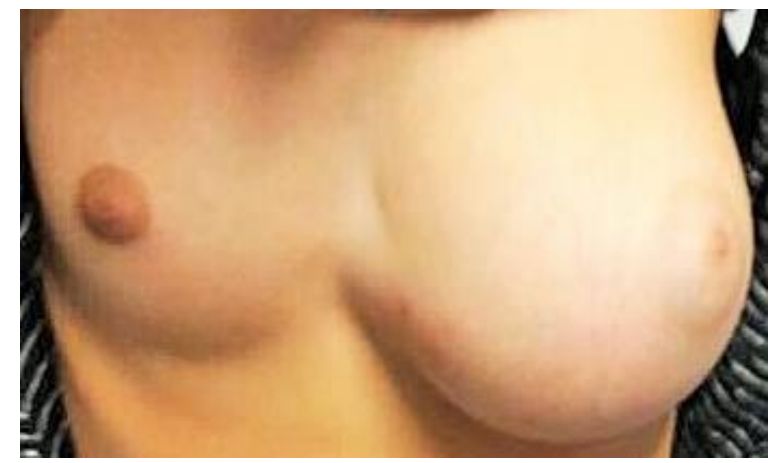

Figura 1. Apresentação do volumoso tumor durante a inspeção mamária na primeira consulta. 


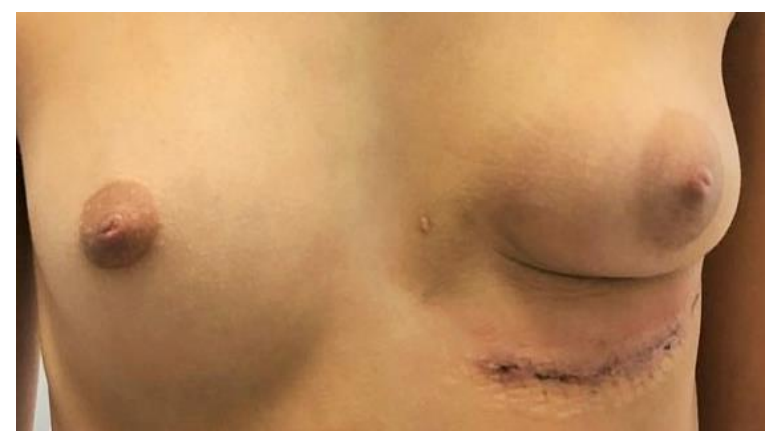

Figura 2. Pós-operatório de seis dias.

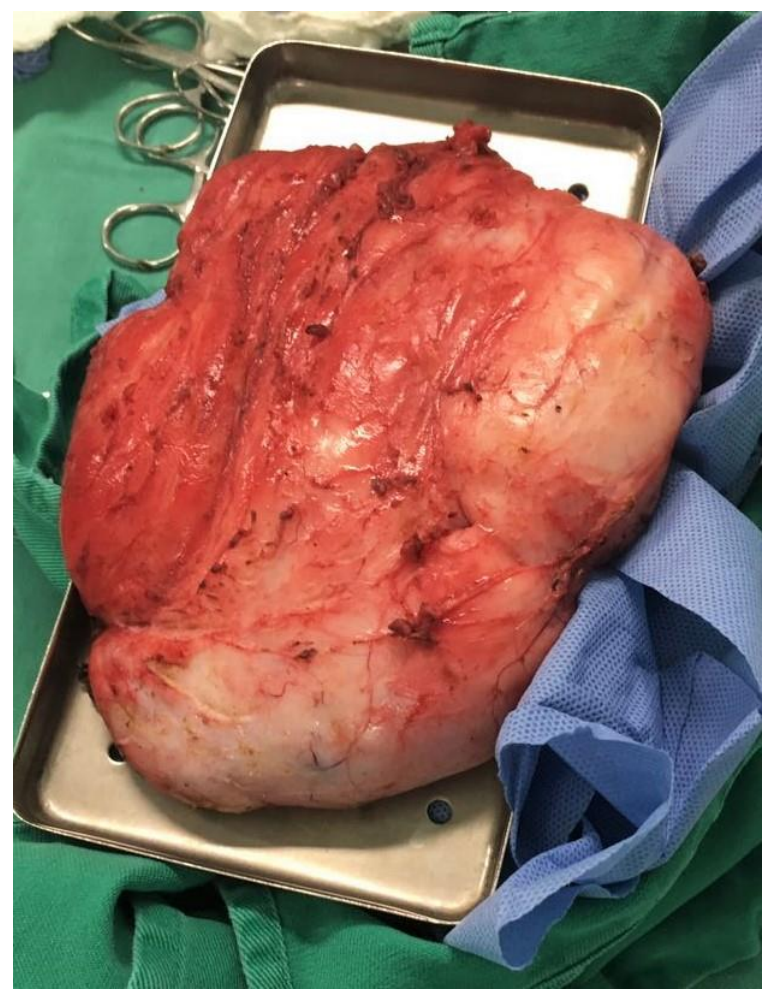

Figura 3. Tumoração com peso de $530 \mathrm{~g}$, dimensões de $14 \times 12 \times 7,5 \mathrm{~cm}$, com superfície externa lobulada, de coloração castanho clara.

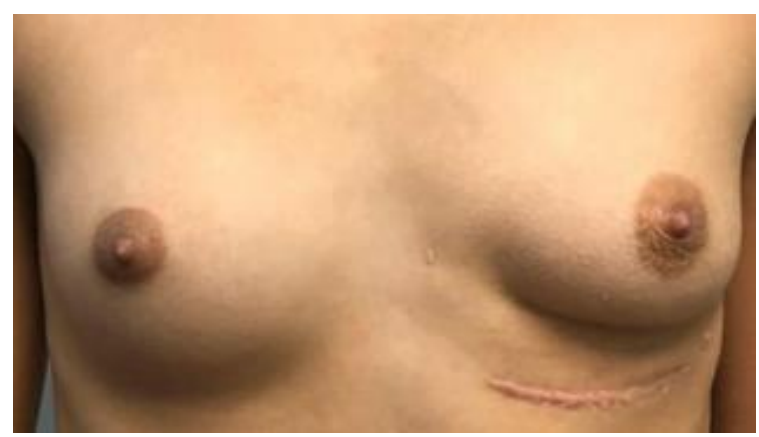

Figura 4. Foto após um ano da tumorectomia.

\section{DISCUSSÃO}

O fibroadenoma juvenil gigante é um tipo de fibroadenoma raro, correspondendo apenas a $4 \%$ dos casos. A apresentação gigante do tumor ocorre quando o tamanho for maior que $5 \mathrm{~cm}$ de diâmetro ou o peso maior que $500 \mathrm{~g}$ ou quando ocupa pelo menos $80 \%$ da mama. Dentre as características, esses tipos de tumores são encapsulados e de crescimento rápido ${ }^{7}$. Esta neoplasia benigna decorre de uma desordem do desenvolvimento do tecido mamário ${ }^{11}$. Segundo dados epidemiológicos, o presente caso clínico encontra-se dentro da margem da faixa etária (10 a 20 anos de idade) e da etnia de maior prevalência (afrodescendente). Tipicamente, é unilateral, apresentando ausência de complicações, como lesões por compressão de tecidos adjacentes, deformação da estrutura mamária, retração mamilar e expansão das veias superficiais ${ }^{8}$.

Existe uma falta de diretrizes claras sobre as modalidades diagnósticas e terapêuticas, dessa forma o manejo varia entre mastologistas, obstetras e ginecologistas, pediatras e cirurgiões pediátricos ${ }^{3}$.

Em comparação com casos similares na literatura verificamos a compatibilidade etária (12 anos) muito frequente na manifestação e abordagem do fibroadenoma juvenil gigante ${ }^{7,12,13}$. Além do curso assintomático e lesão expansiva progressiva ${ }^{1,7,12-14}$.

A biópsia pré-operatória deve ser idealmente realizada através da core biopsy (punção por agulha grossa), pois possibilita a análise histológica e imunohistoquímica, favorecendo a diferenciação entre as neoplasias mamárias. Já a PAAF (punção por agulha fina), muito utilizada para nódulos tireoidianos, tem pouca utilidade para conclusão diagnóstica da tumoração mamária, devido análise citológica apresentar escassez de material tumoral ${ }^{15-16}$.

Como os principais diagnósticos diferenciais são outros nódulos benignos, como cisto mamário, tumor filóides, papiloma, lipoma, hamartoma e adenoma, há necessidade de exames complementares para fazer a sua distinção ${ }^{6}$. No caso relatado, foi realizado o exame de ultrassonografia, seguido por biópsia percutânea com anatomopatológico e, posteriormente, biópsia excisional (tumorectomia), assim concluindo o diagnóstico desta neoplasia mamária.

O diagnóstico pré-operatório de tumor filóides altera a conduta cirúrgica, uma vez que 
esta neoplasia necessita de margem de segurança de pelo menos $1 \mathrm{~cm}$ para evitar a recidiva. Vale lembrar que o tumor filóides é subdividido em benigno, borderline e maligno de acordo com seu índice mitótico (até 4, 5-10 e maior que 10, respectivamente), sendo que a variante maligna apresenta mal prognóstico ${ }^{17}$.

O câncer de mama é uma patologia muito temida por toda população, inclusive entre os pais de uma paciente portadora de neoplasia mamária benigna volumosa. A cancerofobia afeta a qualidade de vida da paciente e seus familiares. Todavia, a incidência de câncer de mama em pacientes jovens em 2003 foi de 0,08 casos em 100.00 mulheres, ou seja, muito baixa ${ }^{18}$.

A conduta cirúrgica é a principal terapia nos casos de tumores mamários volumosos, que podem levar a dor em coluna dorsal devido seu peso, assim como desconforto e lesões dérmicas mamárias como isquemia, eczema e ulceração ${ }^{19}$.

Após a extirpação nodular, o seguimento da paciente portadora de fibroadenoma juvenil gigante submetida à tumorectomia deve ser realizado em longo prazo, afim de reportar os casos de recidiva tumoral e programar novo tratamento cirúrgico, quando necessário ${ }^{20}$.

Com efeito, a opção pela extirpação cirúrgica tumoral é a melhor opção terapêutica, tanto para o diagnóstico quanto para o tratamento, pelo fato da necessidade da avaliação de toda a extensão tumoral pelo patologista $^{3}$. Segundo Sosin et al. ${ }^{3}$, o pósoperatório da tumorectomia do fibroadenoma juvenil gigante apresenta altas taxas de sucesso.

\section{CONCLUSÃO}

Na apresentação de uma massa mamária gigante em pacientes pediátricos/hebiátricos, deve-se considerar o fibroadenoma juvenil gigante como um possível diagnóstico diferencial. O estudo é importante para a comunidade médica, pois se trata de uma neoplasia rara, com evolução rápida, que mimetiza outras neoplasias mamárias. Assim, visa despertar a necessidade de maior atenção para quadros de desenvolvimento tumoral mamário para diferenciação entre histologias benignas e malignas, assim como o estabelecimento de orientações e condutas às pacientes portadoras desta entidade patológica.

\section{DECLARAÇÃO DE CONFLITO DE INTERESSE}

Os autores declaram não haver qualquer potencial conflito de interesse que possa interferir na imparcialidade deste trabalho científico.

\section{REFERÊNCIAS}

1. Al Ghamdi S, Abdullah GA, Safi NA, Rasheed K, Yousef $Y$. Giant juvenile fibroadenoma of breast in adolescent girls. JPS Case Reports. 2018;28:336.

https://doi.org/10.1016/j.epsc.2017.09.029

DOI:

2. Guerra IM, Núñez $A Q$, Sánchez $D R$. Fibroadenoma gigante en una adolescente. MEDISAN. 2014;18(4):569-74.

3. Sosin $M$, Pulcrano $M$, Feldman ED, Patel KM, Nahabedian MY, Weissler JM et al. Giant juvenile fibroadenoma: a systematic review with diagnostic and treatment recommendations. Gland Surg 2015;4:312-21. DOI: https://doi.org/10.1097/MD.0000000000010765

4. García-Rodríguez $R$, Nieto $M A$, Jiménez $C$, Labao L, Hernández-Vicente G. Fibroadenoma gigante juvenil. Clin Invest Ginecol Obstet. 2008;35(6):221-4.

DOI: https://doi.org/10.1016/S0210-573X(08)75108-6

5. Celik SU, Celik DB, Yetiskin E, Ergun E, Percinel $S$, Demirer S. Giant juvenile fibroadenoma of the breast: a clinical case. Arch Argent Pediatr. 2017;115(6):e428-e431.

DOI: https://doi.org/10.5546/aap.2017.eng.e428

6. Nazário ACP, Rego MF, Oliveira VM. Nódulos benignos da mama: uma revisão dos diagnósticos diferenciais e conduta. Rev Bras Ginecol Obstet. 2007;29(4):211-9. DOI: https://doi.org/10.1590/S0100$\underline{72032007000400008 .}$.

7. Gkali C, Giannos A, Primetis E, Chalazonitis A, Anastasiadi T, Feida E. Giant juvenile fibroadenoma in a 12-Year-old girl: ultrasonography, elastography, clinical, and pathology findings of this rare type of fibroadenoma. Ultrasound $Q$. 2017;33(1):51-4. DOI: https://doi.org/10.1097/RUQ.000000000000028 $\underline{3}$

8. Vieira ARA. Terapêutica minimamente invasiva como alternativa à nodulectomia no tratamento do fibroadenoma. [Tese]. Instituto de Ciências Biomédicas Abel Salazar, Universidade do Porto, Porto, 2013. 
9. King KS, Harrington MA, Kassira N. Recurrent giant juvenile fibroadenoma. JPS Case Reports. 2017;26:42-5.

DOI:

https://doi.org/10.1016/i.epsc.2017.08.016

10. Ciftci I, Sekmenli T, Ozbek S, Karamese M, Ugras S. Inframammarial giant fibroadenoma removing and a nipple-sparing breast reconstruction in an adolescent: a Case report. Prague Med Rep. 2015;116(2):161-6. DOI: https://doi.org/10.14712/23362936.2015.54

11. Yagnik VD. Juvenile giant fibroadenoma. Clin Pract. 2011;1(3):2011.e49 DOI: https://doi.org/10.4081/cp.2011.e49

12. Firdaus CM, Norjazliney AJ. Rashid NFA. A case report of juvenile giant fibroadenoma of the breast: How common?. Tzu-Chi Med J. 2017; 29(3):177.

DOI:

https://doi.org/10.4103/tcmj.tcmj 6417

13. Giannos A, Stavrou S, Gkali C, Chra E, Marinopoulos S, Chalazonitis A, Drakakis P. A prepubertal giant juvenile fibroadenoma in a 12year-old girl: Case report and brief literature review. Int J Surg Case Rep. 2017;41:427-30. DOI: https://doi.org/10.1016/j.ijscr.2017.11.026

14. Islam S, Saroop S, Bheem V, Naraynsingh V. Largest giant juvenile fibroadenoma of the breast. BMJ Case Rep. 2019;12(1):e227277. DOI: https://doi.org/10.1136/bcr-2018-227277

15. Kapila K, pathan sK, al-Mosawy Fa, et al. Fine needle aspiration cytology of breast masses in children and adolescents: experience with 1404 aspirates. Acta Cytol 2008;52:681-6. DOI: https://doi.org/10.1159/000325621

16. Homesh $\mathrm{Na}$, Issa $\mathrm{Ma}$, el-sofiani $\mathrm{Ha}$. the diagnostic accuracy of fine needle aspiration cytology versus core needle biopsy for palpable breast lump(s). Saudi Med J. 2005;26:42-6.

17. Gomez CAO, Herazo F, Gil M, Echeverry C, Angel $G$, Borrero $M$, Madrid J, Jaramillo $R$. Phyllodes tumor of the breast: a clinic-pathologic study of 77 cases in a Hispanic cohort. Colombia Med. 2015;46(3):104-8. DOI: https://doi.org/10.25100/cm.v46i3.1595
18. Gutierrez JC, Housri N, Koniaris LG, et al: Malignant breast cancer in children: a review of 75 patients. J Surg Res 2008;147:182-8. DOI: https://doi.org/10.1016/i.jss.2008.03.026

19. Ng WK, Mrad MA, Brown MH. Juvenile fibroadenoma of the breast: treatment and literature review. Can J Plast Surg. 2011; 19(3):105-7.

DOI: https://doi.org/10.1177/229255031101900308

20. Huang IC, Li $p C$, Ding DC. recurrent juvenile fibroadenoma of the breast in an adolescent: a case report. Medicine. 2018;97:e10765. DOI: https://doi.org/10.1097/MD.0000000000010765 